\title{
Etiology analysis of lower gastrointestinal bleeding in children
}

yun liu ( $\nabla$ yunliull@163.com )

JiangXi Provinal Children's Hospital

\section{XiuWen Chen}

JiangXi Provincial Children's Hospital

\section{ShengHua Wan}

JiangXi Provincial Children's Hospital

\section{Research article}

Keywords: lower gastrointestinal bleeding, children, etiology, intussusception

Posted Date: September 17th, 2019

DOI: https://doi.org/10.21203/rs.2.14539/v1

License: (c) (1) This work is licensed under a Creative Commons Attribution 4.0 International License. Read Full License 


\section{Abstract}

Background: In a clinical setting, the common causes of lower gastrointestinal bleeding are often easy to identify, but some cases require detailed examinations to make a diagnosis. At present, the cause of lower gastrointestinal bleeding is unclear. The aim of this study was to review cases of children hospitalized for lower gastrointestinal bleeding in the past 5 years at our hospital and analyze the etiologies according to age group.

Methods: In this study, we statistically analyzed the etiologies of lower gastrointestinal bleeding in children of different ages and sexes from Jiangxi Province, China. From January 2013 to August 2018, 8,036 patients hospitalized for lower gastrointestinal bleeding in Jiangxi Provincial Children's Hospital were divided into groups to analyze the etiologies.

Results: Of these 8,036 patients, 7,827 were clearly diagnosed. Etiologies included intussusception (4,741 cases, $60.6 \%)$, infectious diarrhea (2,171 cases, $27.7 \%)$, colon polyps (405 cases, $5.2 \%)$, Meckel's diverticulum (292 cases, $3.7 \%$ ), allergic colitis (113 cases, $1.4 \%$ ), anal fissures (34 cases, $0.4 \%$ ), HenochSchönlein purpura ( 27 cases, $0.3 \%$ ), eosinophilic gastrointestinal disease ( 27 cases, $0.3 \%$ ), and inflammatory bowel disease (17 cases, $0.2 \%$ ). Generally, the condition presents with blood in the stool, bloody diarrhea, or fecal occult blood. Abdominal color Doppler ultrasonography, colonoscopy, computed tomography, $99 \mathrm{~m}$ Tc pertechnetate imaging, and other blood tests can aid in the diagnosis.

Conclusions: We found that the type of lower gastrointestinal bleeding in children is related to age. Intussusception was the most common cause of intestinal obstruction overall and in infants between 6 and 36 months of age. Treatment of children with lower gastrointestinal bleeding should be based on prevention. Medical staff should actively raise awareness of the condition, identify the severity of the disease in a timely manner, make early diagnoses, provide prompt treatment, and cooperate with providers in multiple disciplines to save patients' lives. Keywords: lower gastrointestinal bleeding, children, etiology, intussusception

\section{Background}

Lower gastrointestinal bleeding (LGIB) is commonly encountered in infants and children in clinical practice; however, its epidemiology has not been well studied [1].LGIB refers to bleeding distal to the ligament of Treitz and is sometimes subcategorized as bleeding from the small bowel (termed mid-GI bleeding) or bleeding from the colon. The likely causes of LGIB vary depending on age. Some patients present with a large amount of bleeding. In cases such as these, a patient's condition changes rapidly and may involve fatal hemorrhagic shock. Other patients display no obvious clinical symptoms, and the condition is only manifested as fecal occult blood; the repeated small amounts of bleeding in these cases may also lead to anemia. Milk-protein-induced allergic colitis, which is associated with long-term repeated blood in the stool, can lead to malabsorption, hypoproteinemia, slow growth, and stunting. It is important for doctors to make the right diagnosis to ensure that appropriate treatment is used [2]. At present, the 
cause of LGIB is unclear. Our lack of understanding may be related to the small sample sizes of previous analyses. The aim of this study was to review the 8,036 cases of children hospitalized for LGIB in the past 5 years at our hospital and analyze the etiologies according to age group.

\section{Methods}

\section{Data source}

From January 2013 to August 2018, 8,036 children were hospitalized for LGIB at Jiangxi Provincial Children's Hospital in Nanchang, China. Among these, 7,827 were clearly diagnosed (5,431 males, 2,396 females). The patients were divided into four age groups: 28 days to 1 year (mean age: $7.3 \pm 3.0$ months), $1-3$ years (mean age $20.6 \pm 6.9$ months), $3-6$ years (mean age $51.6 \pm 10.2$ months), and $>6$ years (mean age $107.9 \pm 26.7$ months). The course of disease varied from 1 to 22 days (average 6.2 days). All children were admitted to the hospital with hematochezia as the primary symptom. The wards involved in treatment included the digestive medical wards, nephrology wards, infectious wards, surgical wards, and hematology wards.

\section{Methods}

We first excluded cases involving suspected foods or medications that can cause false positives for bloody stools. Finally, 7,827 cases were included in the analysis. The cases were grouped by patient age to allow us to summarize the etiologies of LGIB in different age groups. The main causes of LGIB in the same age group were analyzed, and the etiologies of LGIB and distribution of the same disease were compared among age groups. The study design was approved by our hospital's ethics committee.

\section{Statistical analysis}

All data were statistically analyzed using SPSS Statistics version 13.0. Count data were expressed using the sample composition ratio (\%); measurement data were expressed as the mean \pm standard deviation. The multiple sample rates and multiple composition ratios were compared using the chi-squared test. Pvalues less than 0.05 were considered statistically significant.

\section{Results}

In this study, there were 8,036 patients with LGIB, of whom 7,827 were clearly diagnosed and included in the analysis. As shown in Table 1, these included 5,431 (69.4\%) male patients and 2,396 (30.6\%) female patients. As shown in Table 2, 50.3\% of the patients aged 28 days to 1 year, $34.1 \%$ from 1 to 3 years, $10.7 \%$ from 3 to 6 years, and $4.9 \%$ were older than 6 years. Among the 7,827 cases, the distribution was as follows (Table 3): intussusception (4,741 cases, 60.6\%), infectious diarrhea (2,171 cases, $27.7 \%)$, colon polyps (405 cases, 5.2\%), Meckel's diverticulum (292 cases, $3.7 \%$ ), allergic colitis (113 cases, $1.4 \%$ ), 
anal fissures (34 cases, 0.4\%), Henoch-Schönlein purpura (HSP) (27 cases, 0.3\%), eosinophilic gastrointestinal disease (27 cases, $0.3 \%$ ), and inflammatory bowel disease (IBD) (17 cases, $0.2 \%$ ). Table 4 shows the diagnostic distribution among different age groups. Intussusception diagnoses were concentrated in the groups of patients aged 28 days to 1 year $(2,502$ cases, $52.8 \%)$ and 1 to 3 years $(1,775$ cases, $37.4 \%)$. Colon polyps diagnoses were distributed in the 1-to-3-years group (67 cases, $16.5 \%$ ), the 3-to-6-years group (229 cases, 56.5\%), and the >6-years group (109 cases, $26.9 \%$ ). Allergic colitis was diagnosed mainly in the 28-days-to-1-year group (91 cases, $80.5 \%$ ). Infectious diarrhea was diagnosed mainly in the 28-days-to-1-year (1,288 cases, 59.3\%) and 1-to-3-years (732 cases, 33.7\%) groups. HSP was diagnosed in 12 cases $(44.4 \%)$ in both the 3-to-6-years and $>6$-years groups. The distribution of anal fissure in the 4 age groups was 7 cases (20.6\%), 8 cases (23.5\%), 12 cases (35.3\%), and 7 cases $(20.6 \%)$, respectively. IBD was diagnosed in the 3-to-6-years group (8 cases, $47.1 \%$ ) and in the $>6$-years group ( 6 cases, $35.2 \%$ ). The distribution of Meckel's diverticulum in the 4 age groups was 45 cases (15.4\%), 67 cases (22.9\%), 67 cases $(22.9 \%)$, and 113 cases (38.7\%), respectively. Eosinophilic gastrointestinal disease was distributed mainly in the $>6$-years group (15 cases, $55.6 \%$ ). Results of the chi-squared test revealed significant differences in the etiologies of different age groups.

\section{Discussion}

In this study, intussusception was the most common cause of lower gastrointestinal bleeding in children overall and in infants between 6 and 36 months of age. Other etiologies included infectious diarrhea, colon polyps, Meckel's diverticulum, allergic colitis, anal fissures, Henoch-Schönlein purpura, eosinophilic gastrointestinal disease, and inflammatory bowel disease.

The likely causes of LGIB vary depending on the region as well as age. In the United States, the most common causes of rectal bleeding in infants are anal fissure and cow's-milk- or soy-protein-induced colitis. In children 12 months and older, the most common causes of rectal bleeding are infectious gastroenteritis and anal fissures. In each age group, there are other less-common disorders that are also important to identify, as they may be life-threatening and/or require specific treatment. The spectrum of causes varies in other parts of the world [3]. In a report from India, for example, $24 \%$ of 85 children bled from amoebic ulcers [4].

In the present study, intussusception was the most common cause of LGIB, accounting for $60.6 \%$ of the total number of cases. It was also the most common cause of intestinal obstruction in infants between 6 and 36 months of age. Approximately $60 \%$ of affected children were younger than 1 year, and $80 \%$ were younger than 2 years. In this age group, intussusception was usually idiopathic and occurred in the ileocecal region. This is in contrast to older children, in whom a polyp, Meckel's diverticulum, or other lesions often served as a lead point [5]. In general, patients with intussusception present with symptoms of hematochezia. If there are no indications of acute abdomen, an ultrasound-guided saline enema can be administered within 24 hours after onset. If the course of the disease exceeds 48 hours, the child's mental state is poor, symptoms of peritoneal inflammation are observed, there are multiple recurrences, or highly suspected organic lesions induce intussusception, enema reduction should be used with caution, 
as these cases generally require surgery [6]. In this study, 5 cases of intussusception were caused by Meckel's diverticulum, 6 cases were caused by colon polyps, 4 cases were caused by Burkitt's lymphoma, 1 case was caused by intestinal cyst, and 1 case was caused by repeated intestinal malformation.

Pediatric colon polyps are basically juvenile polyps, which are benign hamartomas. They typically occur between the ages of 2 and 10 years, with a peak from 3 to 4 years. Patients usually present with painless rectal bleeding, with or without mucus; a few may have lower abdominal pain from traction on the polyp. Juvenile polyps tend to be pedunculated rather than sessile, and they may autoamputate, which results in significant bleeding [7]. More than $80 \%$ of children with colon polyps have only one or two juvenile polyps. Although they are generally single, benign hamartomas, recurrence occurs in $16.7 \%$ of patients with a single juvenile polyp. A previous study showed that among 23 patients with multiple polyps, 10 had mothers with germline DNA abnormalities in SMAD4, BMPR1A, and PTEN. It is possible to detect related mutations in children with juvenile polyps and predict whether polyps will recur [8]. Although the polyps themselves are not premalignant in those suffering from familial juvenile polyposis, juvenile polyp syndrome, or Peutz-Jeghers syndrome, the syndromes are associated with a higher lifetime risk of colorectal malignancy. These patients should undergo colonoscopies and biopsies every 2 or 3 years to screen for colorectal neoplasia [9].

Colonoscopy is the best way to diagnose colon polyps. During the procedure, polyps can be immediately and painlessly removed. For safety, all polyps are removed for pathological examination to confirm that they are juvenile hamartomas rather than adenomas. In this study, colon polyps accounted for $5.2 \%$ of the total number of cases, and they were mainly found in children older than 3 years.

Allergic colitis is a common cause of the occurrence of blood in the stool of infants. It is an inflammatory reaction caused by the ingestion of cow's-milk or soy proteins. It can occur in infants who are formulafed, or, less commonly, in infants who are breastfed due to cow's milk in the mother's diet. Affected infants have loose stools, usually accompanied by occult or major bleeding, but are otherwise healthy. The condition can be diagnosed using food avoidance and a stimulating test. Treatment involves meticulous elimination of the causative protein from the mothers' diet if the infant is breastfed, or use of a casein-hydrolysate formula. About $10 \%$ of infants are sensitive to casein-hydrolysate formula and require an amino-acid-based formula. The intolerance usually resolves by 18 months of age, at which time an unrestricted diet can be resumed [10]. In this study, allergic colitis accounted for $1.4 \%$ of the total number of cases, mainly concentrated in children younger than 1 year (80.5\%). Most of these patients were cured through avoidance of the trigger foods.

In this study, infectious diarrhea accounted for $27.7 \%$ of the total number of cases. Infectious colitis can be caused by bacterial or viral infections, which lead to blood in the stool. Some patients presented with bloody stools, some with mucus and blood, and some with traces of blood. In cases of bacterial infection, the stool usually has an obvious odor and is accompanied by abdominal pain, urgency, fever, and other symptoms. Salmonella, Shigella, Campylobacter, Escherichia coli 0157:H7, and Clostridium difficile are the most common bacteria that can cause LGIB in preschool children. Clinically, viral infections that 
cause bloody stools are mainly adenoviruses. Cytomegalovirus infection may cause chronic colitis in patients with immunodeficiency, especially in those with IBD or parasitic infections such as amoebic dysentery [11].

HSP is a systemic vasculitis of unknown etiology characterized by marked purpura, abdominal pain, and joint pain. It is primarily a childhood disease that occurs between the ages of 3 and 15 years. Up to $50 \%$ of patients have gastrointestinal symptoms, but only $17.6 \%$ have gastrointestinal bleeding. Gastrointestinal bleeding in HSP patients is due to intussusception or to mucosal damage caused by purpuric lesions of the gastrointestinal tract [12]. In this study, HSP accounted for $0.3 \%$ of the total number of cases and was mainly concentrated in children older than 3 years.

Anal fissures are the most common cause of rectal bleeding in patients younger than 1 year and are also common in older children. Anal fissures in infants often present with painful defecation: straining, grunting, leg stiffening or back arching consistent with withholding behavior, and streaks of bright-red blood on the surface of the stools. Generally, anal fissures are associated with diarrhea and constipation [13]. They are easily diagnosed by spreading the perineal skin to evert the anal canal. Anal-fissure patients are usually treated in the outpatient department. The condition is not life-threatening and usually does not require hospitalization. One limitation to our study was that, as all included cases were hospitalized, the number of simple anal-fissure cases was small.

IBD is composed of two major disorders: ulcerative colitis (UC), which affects only the colon, and Crohn disease (CD), which can involve any portion of the gastrointestinal tract. UC involves a general and persistent lesion limited to mucosal inflammation. $C D$ is characterized by transmural inflammation and segmental lesions. The transmural inflammation may lead to fibrosis and stenosis, as well as obstructive clinical manifestations that are often absent in UC. More commonly, transmural inflammation leads to sinuses, causing micro-perforations and fistulas [14]. Approximately $40 \%$ of $C D$ patients and approximately $80 \%$ of UC patients present with visible rectal bleeding. The incidence of IBD peaks in late adolescence and early adulthood. Many IBD patients have iron deficiency and/or an elevated erythrocyte sedimentation rate (ESR) or C-reactive protein (CRP), hypoalbuminemia, and elevated fecal calprotectin. The CRP is elevated in $100 \%$ of CD patients and in $60 \%$ of UC patients, and ESR is elevated in $85 \%$ of CD patients and in $23 \%$ of UC patients [14]. Fecal calprotectin $>200 \mathrm{mcg} / \mathrm{g}$ indicates a high possibility of IBD; the diagnosis is confirmed using combinations of gastroscopy, colonoscopy, and biopsy $[15,16]$. Adults and children with IBD may have similar clinical features; however, children have unique complications, including growth disorders and delayed puberty [17]. IBD accounted for $0.2 \%$ of the cases in this study. According to previous research, the prevalences of UC and CD in China are 11.6 cases per 100,000 people and 1.4 cases per 100,000 people, respectively. However, the number of IBD patients increased by 2.11 in 2001 and by 2.78 in 2003 . There are also reports that the number of UC patients in Hong Kong has increased by more than 6 times in the past 20 years [18].

The spectrum of diseases in China is changing. It is important for doctors to constantly update their knowledge, improve their understanding of the disease, and provide accurate diagnoses and treatment in 
a timely manner.

In this study, Meckel's diverticulum accounted for $3.7 \%$ of the total number of cases. There were 67 cases in both the 3-to-6-years group and the 1-to-3-years group. There were no significant differences between the two groups, but the number of cases in the >6-years group was significantly higher than in the other groups (113 cases). Meckel's diverticulum results from incomplete obliteration of the omphalomesenteric duct. It is usually asymptomatic but may cause painless rectal bleeding. This bleeding may be chronic and insidious or acute and massive. It is often caused by mucosal ulceration of adjacent small-bowel tissue due to production of acid by ectopic gastric tissue within the diverticulum. Other complications associated with Meckel's diverticulum include obstruction, perforation, diverticulitis, and intussusception. Approximately $60 \%$ of pediatric patients with complications from Meckel's diverticulum are younger than 2 years of age19]. In general, Meckel's diverticulum is associated with acute bleeding of large amounts, moderate-to-severe anemia, and perfect blood tests. Color Doppler ultrasonography, 99m Tc pertechnetate, and other tests can help diagnose the condition. Clinicians must ensure prompt diagnosis and treatment and consult a surgeon in time to avoid life-threatening shock. If a Meckel's diverticulum diagnosis is considered, a surgeon should be immediately contacted.

Eosinophilic gastroenteritis accounted for $0.3 \%$ of the total number of cases in this study. It is an immune-mediated inflammatory disease characterized by localized or diffused eosinophil infiltration in various parts of the digestive tract. Children mainly present with abdominal pain: mostly paroxysmal umbilical or upper abdominal pain with diarrhea or blood in the stool. Blood indicates that the lesion involves the colon. Diagnosis of eosinophilic gastroenteritis requires a combination of medical history and endoscopy. Pathology results show that $50 \%$ of patients with eosinophilic gastroenteritis have food allergies, and an avoidance allergy diet and anti-allergy treatments are effective [20].

In this study, six patients with blood in the stool had non-digestive diseases. One infant was diagnosed with late-onset vitamin K1 deficiency, and five patients were diagnosed with thrombocytopenia. Abnormal coagulation may cause gastrointestinal bleeding, but there are also skin-bleeding spots or ecchymoses. The possibility of abnormal coagulation can be identified by combining clinical history, physical examinations, and blood tests.

Of the children included in this study, 202 with LGIB underwent abdominal color Doppler ultrasonography, colonoscopy, and blood tests. The reasons for these tests are not clear, and follow-up is ongoing. One patient had blood in the stool due to unexplained varicose veins.

This study has revealed that we can preliminarily judge the cause of LGIB according to a child's medical history, age, and other available information, then do a corresponding examination to confirm the diagnosis and reduce unnecessary treatment.

\section{Conclusions}


Treatment of children with LGIB should be based on prevention. Medical staff should actively raise awareness of the condition, identify the severity of the disease in a timely manner, make early diagnoses, provide prompt treatment, and cooperate with providers in multiple disciplines to save patients' lives. For some occult diseases, blood in the stool serves as a clue, and actively looking for the cause, early diagnosis, and early treatment do not affect the growth and development of the child.

\section{Abbreviations}

C-reactive protein (CRP), Crohn disease (CD), erythrocyte sedimentation rate (ESR), Henoch-Schönlein purpura (HSP), inflammatory bowel disease (IBD), lower gastrointestinal bleeding (LGIB), ulcerative colitis (UC)

\section{Declarations}

- Ethics approval and consent to participate: The study was approved by the appropriate hospital ethics committee. The requirement for informed consent was waived. The full name of the ethics committee: JiangXi Provincial Children's Hospital Medical Ethics Committee. the committee's reference number:JXSETYY-YXKY-20190084.

- Consent for publication:

- Competing interest: The authors declare that they have no competing interests.

- Availability of data and materials: All data generated or analysed during this study are included in this published article.

- Author contributions: YL carried out the experiment. YL wrote the manuscript with support from XC. YL and SW conceived the original idea. All authors have read and approved the manuscript.

- Acknowledgements: Not Applicable.

- Funding: No funding was obtained for this study.

\section{References}

1. Pant C, Olyaee M, Sferra TJ, Gilroy R, Almadhoun O, Deshpande A (2015) Emergency department visits for gastrointestinal bleeding in children: results from the Nationwide Emergency Department Sample 2006-2011. Curr Med Res Opin 31:347.

2. Rayhorn N, Thrall C, Silber G (2001) A review of the causes of lower gastrointestinal tract bleeding in children. Gastroenterol Nurs 24:77-82.

3. Teach SJ, Fleisher GR (1994) Rectal bleeding in the pediatric emergency department. Ann Emerg Med 23:1252.

4. Khurana AK, Saraya A, Jain N, Chandra M, Kulshreshta R (1998) Profile of lower gastrointestinal bleeding in children from a tropical country. Trop Gastroenterol 19:70. 
5. Ntoulia A, Tharakan SJ, Reid JR, Mahboubi S (2016) Failed intussusception reduction in children: correlation between radiologic, surgical, and pathologic findings. AJR Am J Roentgenol 207:424.

6. Flaum V, Schneider A, Gomes Ferreira C, Philippe P, Sebastia Sancho C, Lacreuse I, Moog R, Kauffmann I, Koob M, Christmann D, Douzal V, Lefebvre F, Becmeur F (2016) Twenty years' experience for reduction of ileocolic intussusceptions by saline enema under sonography control. $J$ Pediatr Surg 51(1):179-182.

7. Lee BG, Shin SH, Lee YA, Wi JH, Lee YJ, Park JH (2012) Juvenile polyp and colonoscopic polypectomy in childhood. Pediatr Gastroenterol Hepatol Nutr 15:250.

8. Fox VL, Perros S, Jiang H, Goldsmith JD (2010) Juvenile polyps: recurrence in patients with multiple and solitary polyps. Clin Gastroenterol Hepatol 8:795.

9. Tavusbay C, Acar T, Kar H, Atahan K, Kamer E (2018) The patients with Peutz-Jeghers syndrome have a high risk of developing cancer. Turk J Surg. 34(2):162-164.

10. Odze RD, Wershil BK, Leichtner AM, Antonioli DA (1995) Allergic colitis in infants. J Pediatr 126:163.

11. Huilan S, Zhen LG, Mathan MM, Mathew MM, Olarte J, Espejo R, Maung UK, Ghafoor MA, Khan MA, Sami Z (1991) Etiology of acute diarrhoea among children in developing countries: a multicentre study in five countries. Bull World Health Organ 69:549.

12. Chang WL, Yang YH, Lin YT, Chiang BL (2004) Gastrointestinal manifestations in Henoch-Schönlein purpura: a review of 261 patients. Acta Paediatr 93:1427.

13. Jafari SA, Kiani MA, Kianifar HR, Mansooripour M, Heidari E, Khalesi M (2018) Etiology of gastrointestinal bleeding in children referred to pediatric wards of Mashhad hospitals, Iran. Electron Physician 10(2): 6341-6345.

14. Silverberg MS, Satsangi J, Ahmad T, et al. (2005) Toward an integrated clinical, molecular and serological classification of inflammatory bowel disease: report of a Working Party of the 2005 Montreal World Congress of Gastroenterology. Can J Gastroenterol 19 Suppl A:5A.

15. Limburg PJ, Devens ME, Harrington JJ, DiehI NN, Mahoney DW, Ahlquist DA (2003) Prospective evaluation of fecal calprotectin as a screening biomarker for colorectal neoplasia. Am J Gastroenterol 98:2299.

16. Korman LY, Overholt BF, Box T, Winker CK (2003) Perforation during colonoscopy in endoscopic ambulatory surgical centers. Gastrointest Endosc 58:554.

17. Heuschkel R, Salvestrini C, Beattie RM, Hildebrand H, Walters T, Griffiths A (2008) Guidelines for the management of growth failure in childhood inflammatory bowel disease. Inflamm Bowel Dis 14:839.

18. Ye L, Cao Q, Cheng J (2013) Review of Inflammatory Bowel Disease in China. Scientific World Journal 2013:296470.

19. Glenn IC, El-Shafy IA, Bruns NE, Muenks EP, Duran YK, Hill JA, Peter SDS, Prince JM, Lipskar AM, Ponsky TA (2018) Simple diverticulectomy is adequate for management of bleeding Meckel diverticulum. Pediatr Surg Int 34(4):451-455. 
20. Khan S, Orenstein SR (2002) Eosinophilic gastroenteritis: epidemiology, diagnosis and management. Paediatr Drugs 4(9):563-570.

\section{Tables}

Table 1. Sex ratio of the included cases

\begin{tabular}{ccc}
\hline Sex & Number of valid cases & Percentage \\
\hline Male & 5431 & 69.4 \\
\hline Female & 2396 & 30.6 \\
Total & 7827 & 100.0 \\
\hline
\end{tabular}

*P-value $<0.05$ by chi-square test

Table 2. Number of cases of lower gastrointestinal bleeding in different age groups

\begin{tabular}{ccc}
\hline Age group & Number of valid cases & Percentage \\
\hline 28 days-1 year & 3935 & 50.3 \\
1-3 years & 2669 & 34.1 \\
\hline 3-6 years & 840 & 10.7 \\
>6 years & 383 & 4.9 \\
\hline Total & 7827 & 100.0 \\
\hline
\end{tabular}

*P-value $<0.05$ by chi-square test 
Table 3. Distribution of the major etiologies of lower gastrointestinal bleeding

\begin{tabular}{lcc}
\hline Diagnosis & Number of valid cases & Percentage \\
\hline 1. Intussusception & 4741 & 60.6 \\
\hline 1. Colon polyps & 405 & 5.2 \\
\hline 1. Allergic colitis & 113 & 1.4 \\
\hline 1. Infectious diarrhea & 2171 & 27.7 \\
\hline 1. Henoch-Schönlein purpura & 27 & .3 \\
\hline 1. Anal fissures & 34 & .4 \\
\hline 1. Inflammatory bowel disease & 17 & .2 \\
\hline 1. Meckel's diverticulum & 292 & 3.7 \\
\hline 1. Eosinophilic gastrointestinal disease & 27 & .3 \\
\hline Total & 7827 & 100.0 \\
\hline
\end{tabular}

Table 4. Distribution of diagnoses among different age groups

\begin{tabular}{lccccc}
\hline \multicolumn{1}{c}{ Diagnosis } & $\begin{array}{c}28 \text { days-1 year } \\
(\%)\end{array}$ & $\begin{array}{c}1-3 \text { years } \\
(\%)\end{array}$ & $\begin{array}{c}3-6 \text { years } \\
(\%)\end{array}$ & $\begin{array}{c}>6 \text { years } \\
(\%)\end{array}$ & Total \\
\hline 1. Intussusception & $2502(52.8)$ & $1775(37.4)$ & $390(8.2)$ & $74(1.5)$ & 4741 \\
\hline 1. Colon polyps & $0(0)$ & $67(16.5)$ & $229(56.5)$ & $109(26.9)$ & 405 \\
\hline 1. Allergic colitis & $91(80.5)$ & $9(7.9)$ & $11(9.7)$ & $2(1.8)$ & 113 \\
\hline 1. Infectious diarrhea & $1288(59.3)$ & $732(33.7)$ & $106(4.9)$ & $45(2.1)$ & 2171 \\
\hline 1. Henoch-Schönlein purpura & $0(0)$ & $3(11.1)$ & $12(44.4)$ & $12(44.4)$ & 27 \\
\hline 1. Anal fissures & $7(20.6)$ & $8(23.5)$ & $12(35.3)$ & $7(20.6)$ & 34 \\
\hline 1. Inflammatory bowel disease & $1(5.9)$ & $2(11.8)$ & $8(47.1)$ & $6(35.2)$ & 17 \\
\hline $\begin{array}{l}\text { 1. Meckel's diverticulum } \\
\text { 1. Eosinophilic gastrointestinal } \\
\text { disease }\end{array}$ & $45(15.4)$ & $67(22.9)$ & $67(22.9)$ & $113(38.7)$ & 292 \\
\hline \multicolumn{1}{c}{ Total } & $1(3.7)$ & $6(22.2)$ & $5(18.5)$ & $15(55.6)$ & 27 \\
\hline
\end{tabular}


Page 12/12 